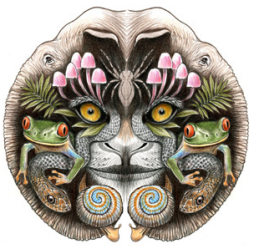

ISSN 0974-7907 (Online) ISSN $0974-7893$ (Print)

OPEN ACCESS

\title{
NeW ReCords of Cerambycidae (INSECTA: Coleoptera) FROM MADHYA PRADESH, INDIA
}

\author{
Amitava Majumder ${ }^{1}$, Angshuman Raha ${ }^{2}$, Bulganin Mitra ${ }^{3} \&$ Kailash Chandra ${ }^{4}$ \\ 1,2,3,4 Zoological Survey of India, Prani Vigyan Bhavan, M- Block, New Alipore, Kolkata, West Bengal 700053, India \\ ${ }^{1}$ amitavamajumder.eco@gmail.com (corresponding author), ${ }^{2}$ adroitangshuman@gmail.com, \\ ${ }^{3}$ bulganinmitra@gmail.com, ${ }^{4}$ kailash611@rediffmail.com
}

Abstract: The present study reports new distribution records of seven species of Cerambycid beetles belonging to seven genera of seven tribes and under two subfamilies from Madhya Pradesh. As a result, based on the past literature and the present study, a total of 18 species of Cerambycidae are known from Madhya Pradesh. The paper also provides detailed diagnostic characters of the eight studied species.

Keywords: Cerambycidae, Coleoptera, invertebrate, longhorn beetles, Madhya Pradesh.

Abbreviations: Coll. - Collected by; ZSI - Zoological Survey of India; L - Length; B- Breadth; FRH - Forest Rest House; Reg. no. - Registration number; Dist. - District.

Wood is an important renewable but easily biodegradable raw material, prone to predation by a large number of insect wood-borers in the logging centres. So, felling of trees in the forests provide abundant food material to the insect wood-borers, which under favorable conditions, multiply rapidly. Deterioration of wood by one of the major wood-borers, Cerambycidae is a chronic phenomenon the world over, more particularly in countries lying within the latitudes of the tropics.

These insects, both in their larval and adult stages usually impact live, dead and dying trees in the forest stands, to felled logs in extraction centres, to timber in storage and in human use, and to creepers.

The longhorn beetles or Cerambycidae are classified together with Chrysomelidae and Bruchidae in the superfamily Chrysomeloidea. The long antennae, absence of a beak, and characteristic tarsi serve to separate most adults of this very large family from other beetles.

The family Cerambycidae currently contains more than 35,700 described species and sub-species in the world and over 1,500 species in India (Norbert Delahaye
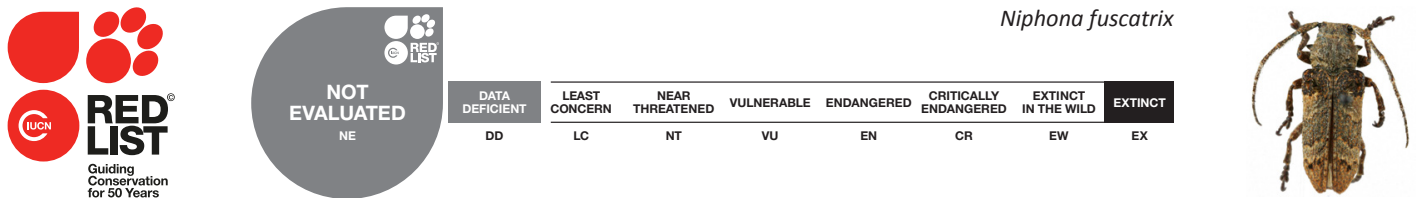

DOI: http://dx.doi.org/10.11609/jott.2465.7.15.8242-8249 | ZooBank: urn:Isid:zoobank.org:pub:E94994D4-3EBB-415E-9A0D-AB4DFE29FA00

Editor: Norbert Delahaye, Colombo, Sri Lanka.

Date of publication: 26 December 2015 (online \& print)

Manuscript details: Ms \# 03969 | Received 16 March 2014 | Final received 16 November 2015 | Finally accepted 28 November 2015

Citation: Majumder, A., A. Raha, B. Mitra \& K. Chandra (2015). New records of Cerambycidae (Insecta: Coleoptera) from Madhya Pradesh, India. Journal of Threatened Taxa 7(15): 8242-8249; http://dx.doi.org/10.11609/jott.2465.7.15.8242-8249

Copyright: (c) Majumder et al. 2015. Creative Commons Attribution 4.0 International License. JoTT allows unrestricted use of this article in any medium, reproduction and distribution by providing adequate credit to the authors and the source of publication.

Funding: Ministry of Environment Forests and Climate Change.

Conflict of Interest: The authors declare no competing interests.
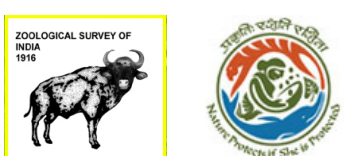

Acknowledgements: Authors are grateful to Dr. K. Venkataraman, Director, ZSI, for providing necessary facilities and encouragements. Thanks are also due to Madhya Pradesh Forest Department for providing necessary permissions and support to carry out the present work. 


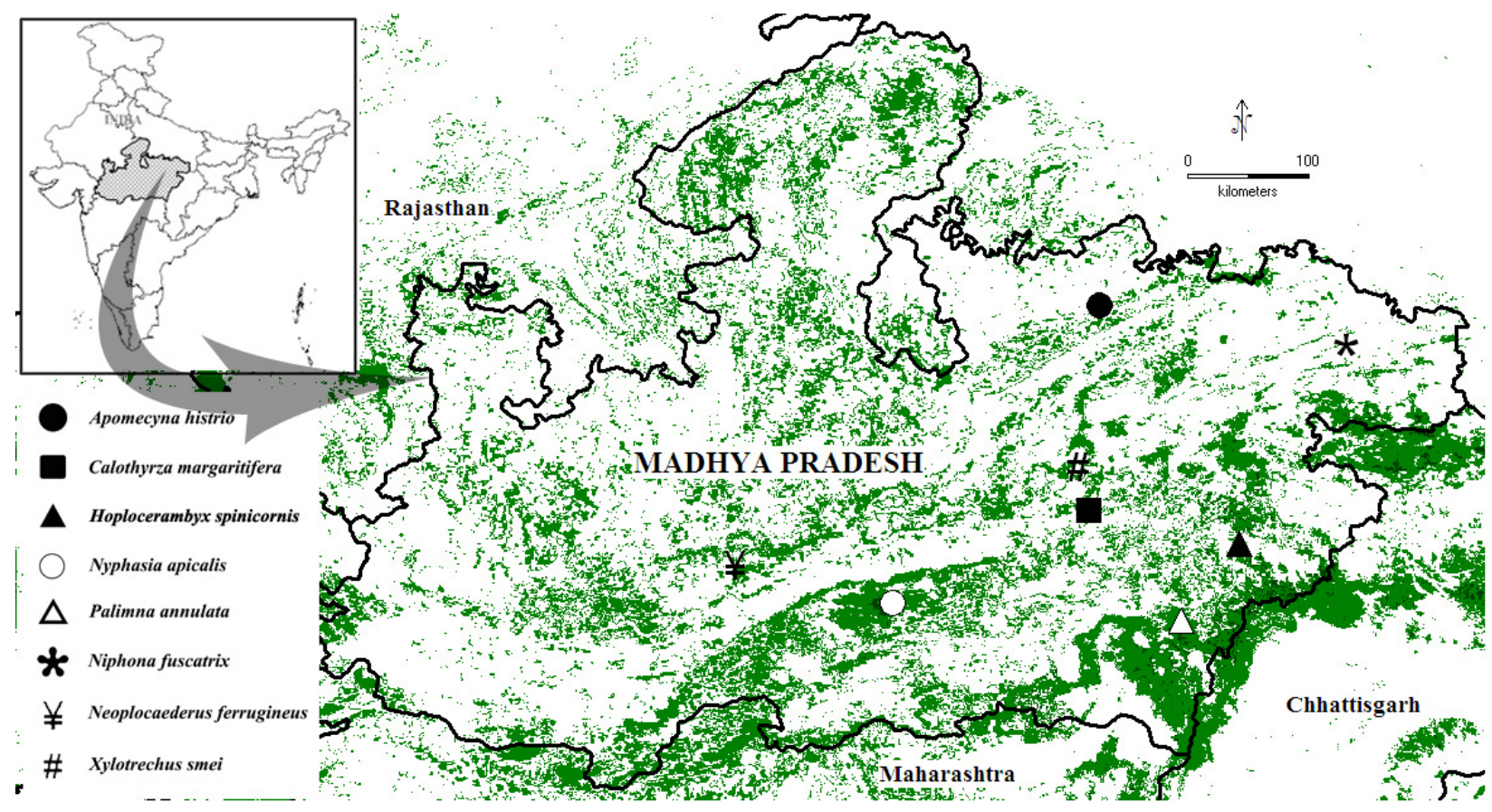

Figure 1. Collection sites of the eight cerambycidae beetles from Madhya Pradesh with the vegetation cover of the state.

pers. comm. 2014). The taxonomic work on Cerambycid beetles of Madhya Pradesh is very scanty. Previously, Chandra et al. (2010) reported 11 species from Madhya Pradesh and presently seven species of longhorn beetles belonging to seven genera of seven tribes under two subfamilies were added to the fauna of Madhya Pradesh (Fig. 1), thus, taking the tally up to 18 species for the state (Table 1). The information on distribution of species was collected from the website of Lamiaires du Monde (2013).

\section{MATERIALS AND METHOdS}

Study period: The specimens were collected from different parts of Madhya Pradesh during field surveys of the Zoological Survey of India from 1999 to 2011.

Study Area: Madhya Pradesh is situated at the center of India covering an area of $308.252 \mathrm{~km}^{2}$ and extending between $21.2^{\circ}-26.87^{\circ} \mathrm{N} \& 74.03^{\circ}-82.81^{\circ} \mathrm{E}$. Forests, including monoculture plantations, cover nearly $94,689 \mathrm{~km}^{2}$ of the stat. The forest types mainly include sub-tropical hill forests, moist deciduous forests, dry deciduous forests and thorny vegetation. Most of the forests are Sal dominated. Major rivers include Chambal, Narmada and Tapti.

Methods: Cerambycid beetles were collected generally at night with the help of a light trap. The coordinates of the collection sites were recorded using GPS, which were further used in preparing maps of the survey sites in DIVA-GIS (Fig. 1; Table 2). Specimens were studied under Leica EZ4 HD binocular microscope for identification. The identified specimens were deposited in the National Zoological Collection, Zoological Survey of India, Kolkata.

\section{RESULTS}

\section{Subfamily: Cerambycinae}

Tribe: Cerambycini

1. Neoplocaederus ferrugineus (Linnaeus) (Image 1A,B) 1758. Cerambyx ferrugineus Linnaeus, Syst. Nat. Laur. Salvius. Holmiae, (10 ${ }^{\text {th }}$ ed.): 390.

1787. Ceramryx gigas Fabricius, Hafniae, Proft 1 : 132. (syn.)

1817. Lamia umbrina Dalman in Schönherr, Scaris, Lewerentziana 1 (3): 161. (syn.)

1853. Hammaticherus nitidus White, Longicornia I, Lond. 7: 127. (syn.)

1859. Cerambyx versutus Pascoe, Trans. Ent. Soc. Lond. (NS) 5: 20. (syn.)

1890. Plocaederus ferrugineus var. niger Gahan, Ann. Mag. Nat. Hist. (6) 5 (25): 51.

1906. Plocaederus ferrugineus; Gahan, Fauna Brit. India, Col. 1: 121.

1991. Neoplocaederus ferrugineus, Sama G. Bollettino della Società Entomologica Italiana. 123(2): 121-128.

Material examined: Reg. no. I 10388, 04.iv.2011, 1 
Table 1. List of known and recorded species of Cerambycidae from Madhya Pradesh. $\left({ }^{*}\right)$ New records from the state.

\begin{tabular}{|c|c|c|}
\hline & Subfamily/Species & Distribution Reference \\
\hline & \multicolumn{2}{|l|}{ Cerambycinae } \\
\hline 1 & $\begin{array}{l}\text { Neoplocaederus pedestris (White, } \\
\text { 1853) }\end{array}$ & Chandra et al. (2010) \\
\hline 2 & $\begin{array}{l}\text { *Neoplocaederus ferrugineus } \\
\text { (Linnaeus, 1758) }\end{array}$ & $\begin{array}{l}\text { Makihara et al. (2008); } \\
\text { Namboodiri \& Thirumalai } \\
\text { (2009) }\end{array}$ \\
\hline 3 & $\begin{array}{l}\text { *Hoplocerambyx spinicornis } \\
\text { (Newman, 1842) }\end{array}$ & $\begin{array}{l}\text { Gahan (1906); Gressitt, } \\
\text { Rondon \& Breuning (1970); } \\
\text { Mukhopadhyay \& Halder } \\
\text { (2003) }\end{array}$ \\
\hline 4 & $\begin{array}{l}\text { *Xylotrechus smei (Laporte \& } \\
\text { Gory, 1841) }\end{array}$ & $\begin{array}{l}\text { Gahan (1906); Basak \& Biswas } \\
\text { (1993); Mukhopadhyay \& } \\
\text { Halder (2003); Ghate (2012) }\end{array}$ \\
\hline 5 & $\begin{array}{l}\text { Stromatium barbatum (Fabricius, } \\
1775 \text { ) }\end{array}$ & Chandra et al. (2010) \\
\hline 6 & Xystrocera globosa (Olivier, 1795) & Chandra et al. (2010) \\
\hline \multirow[t]{2}{*}{7} & $\begin{array}{l}\text { * Stenodryas apicalis (Gahan, } \\
\text { 1893) }\end{array}$ & $\begin{array}{l}\text { Khan \& Khan (1987); Ghate } \\
\text { (2012). }\end{array}$ \\
\hline & \multicolumn{2}{|l|}{ Lamiinae } \\
\hline 8 & Acalolepta nivosa (White, 1858) & Chandra et al. (2010) \\
\hline 9 & Nupserha lenita (Pascoe, 1867) & Chandra et al. (2010) \\
\hline 10 & $\begin{array}{l}\text { Stibara nigricornis (Fabricius, } \\
1781 \text { ) }\end{array}$ & Chandra et al. (2010) \\
\hline 11 & $\begin{array}{l}\text { Calothyrza sehestedii (Fabricius, } \\
\text { 1798) }\end{array}$ & Chandra et al. (2010) \\
\hline 12 & *Palimna annulata (Olivier, 1792) & $\begin{array}{l}\text { Gressitt, Rondon \& Breuning } \\
\text { (1970) }\end{array}$ \\
\hline 13 & $\begin{array}{l}\text { *Apomecyna histrio (Fabricius, } \\
\text { 1793) }\end{array}$ & $\begin{array}{l}\text { Namboodiri \& Thirumalai } \\
\text { (2009) }\end{array}$ \\
\hline 14 & $\begin{array}{l}\text { *Niphona fuscatrix (Fabricius, } \\
1793 \text { ) }\end{array}$ & $\begin{array}{l}\text { Basak \& Biswas (1993); } \\
\text { Namboodiri \& Thirumalai } \\
\text { (2009) }\end{array}$ \\
\hline \multirow[t]{2}{*}{15} & Olenecamptus sp. & Chandra et al. (2010) \\
\hline & \multicolumn{2}{|l|}{ Prioninae } \\
\hline 16 & $\begin{array}{l}\text { Acanthophorus serraticornis } \\
\text { (Olivier, 1795) }\end{array}$ & Chandra et al. (2010) \\
\hline 17 & Macrotomini sp. & Chandra et al. (2010) \\
\hline 18 & $\begin{array}{l}\text { Prionomma atratum (Gmelin, } \\
\text { 1789) }\end{array}$ & Chandra et al. (2010) \\
\hline
\end{tabular}

ex, Bamhori FRH, Singhori Wildlife Sanctuary, Raisen District, coll. S. Sambath.

Diagnostic characters: Medium-sized ( $\mathrm{L}-41 \mathrm{~mm}, \mathrm{~B}$ - $14 \mathrm{~mm}$ ) beetle, elongate, slender, ashy-grey, covered with pale yellowish dense pubescence, pronotum darker, head and legs wholly ashy-grey, head vertical, eyes black, subdivided, upper lobe smaller than lower lobe; antenna 11-segmented, as long as body, pubescent, segments ashy-grey throughout with dark brown apical margin, segment-1 robust, segment-3 little longer than 1 , rest equal in size; pronotum rectangular, ashy-grey with dense pale yellow pubescence, sparsely punctate, medially, deeply incised, surface gibbous, strongly punctate, lateral margins with acute, prominent spines; scutellum small, tongue shaped, brownish in colour; elytra elongated, basally broad, narrowed towards apex, shoulder hump prominent, basal region densely, coarsely, punctate, these gradually finer towards apex, punctation dark brown in colour on the basal region, elytral apex truncated; venter densely pubescent; legs densely pubescent, femur pedunculate, mid and hind tibia apically spined, tarsal claw almost $90^{\circ}$ angle.

Distribution: India: Madhya Pradesh (Raisen) and Tamil Nadu; Sri Lanka, imported in Nigeria.

\section{Hoplocerambyx spinicornis (Newman) (Image 1C,D)}

1842. Hammaticherus spinicornis Newman, Entomologist. 1 (15): 245.

1857. Cerambyx? morosus Pascoe, Trans. Ent. Soc. Lond. (Serie 2) 4: 92. (syn.)

1864. Hoplocerambyx Spinicornis; Thomson, Mém. Soc. Royale Sc. Liège 19: 230.

1866. Hoplocerambyx relictus Pascoe, Proc. Zool. Soc. Lond. : 528. (syn.)

1912. Hoplocerambyx spinicornis; Aurivillius, Coleo. Cat. pars 39 [vol. 22]: 56.

1923. Haplocerambyx minor Pic, L'Échange 39 (412): 8. [misspelling] (syn.)

Table 2. A list of sampling locations along with GPS coordinates

\begin{tabular}{|c|l|l|l|l|l|}
\hline & District & Collection site & Species collected & Latitude & Longitude \\
\hline 1 & Raisen & Singhori WLS; Bamhori RH & Neoplocaederus ferrugineus & $22.750^{\circ} \mathrm{N}$ & $77.250^{\circ} \mathrm{E}$ \\
\hline 2 & Dindori & Chadabajay & Hoplocerambyx spinicornis & $22.950^{\circ} \mathrm{N}$ & $81.083^{\circ} \mathrm{E}$ \\
\hline 3 & Damoh & Veerangana Durgavati WLS; FRH & Xylotrechus smei & $23.502^{\circ} \mathrm{N}$ & $79.850^{\circ} \mathrm{E}$ \\
\hline 4 & Hoshangabad & Panchmarhi BR; Churna FRH & Stenodryas apicalis & $22.466^{\circ} \mathrm{N}$ & $78.433^{\circ} \mathrm{E}$ \\
\hline 5 & Balaghat & Kanha NP; Mukki RH & Palimna annulata & $22.333^{\circ} \mathrm{N}$ & $80.633^{\circ} \mathrm{E}$ \\
\hline 6 & Jabalpur & Madanmahal & Calothyrza sehestedii & $23.166^{\circ} \mathrm{N}$ & $79.933^{\circ} \mathrm{E}$ \\
\hline 7 & Seoni & Panna Tiger Reserve; Karmajhiri & Apomecyna histrio & $24.730^{\circ} \mathrm{N}$ & $80.010^{\circ} \mathrm{E}$ \\
\hline 8 & Sidhi & Mohania & Niphona fuscatrix & $24.416^{\circ} \mathrm{N}$ & $81.883^{\circ} \mathrm{E}$ \\
\hline
\end{tabular}


Material examined: Reg. no. 20091/H4A, 28.vii.2000, 1 ex, Chadabajay, Dist. Dindori, Coll. G.P. Shukla.

Diagnostic characters: Body large (L - 42mm, B - 15mm), dark brown, clothed with fine yellowish pubescence, head elongated, horizontal, mandible well developed, frons small, narrowed towards apex, surface roughened, a deep sulcation in between the antennal base, started from vertex to frons, eyes sub-divided, lower lobe much larger than upper one, vertex finely granulated, sparsely yellowish pubescent; antennae 11- segmented, stout, dark brown, sparsely pubescent, segment- 1 stout, robust, segment- 3 much longer than segment- 4 , segment- 3 onwards apical margin bear acute spine internally; pronotum elongated, globular shaped, surface roughened with distinct ridges, sparsely yellowish pubescence; elytra elongated, finely punctured, sparsely, finely pubescent, surface with few indistinct longitudinal ridges, apex truncated, sutural spine acute; venter dark brown with finely silky pubescence, coxal cavities closed, meso-sternal process apically truncated; legs dark brown, femur elongated, robust, tibia elongated, slender, tarsal claws more than $90^{\circ}$ angle.

Distribution: India: Assam, Madhya Pradesh (Dindori), Meghalaya and Sikkim; Afghanistan, Bhutan, Borneo, Indonesia, Laos, Malaysia, Myanmar, Nepal, Pakistan, Papua New Guinea, Philippines, and Thailand.

\section{Tribe: Clytini}

\section{Xylotrechus smei (Laporte \& Gory) (Image 1E,F)}

1841. Clytus Smei Laporte \& Gory, Hist. Nat. Icon. Ins. Coleo. 37 [female]

1841. Clytus vicinus Laporte \& Gory, Hist. Nat. Icon. Ins. Coleo. 37. [male] (syn.)

1855. Clytus Smeei; White, Longicornia II, Lond. 8: 262 [misspelling]

1906. Xylotrechus smei; Gahan, Fauna Brit. India, Col. 1: 241.

Material examined: 1ex, Veerangana Durgavati Wildlife Sanctuary, Dist. Damoh, FRH, Sing, 23.vi.2009. Coll. J. Tilak. Reg. no. 20092/ H4A.

Diagnostic characters: Small size ( $\mathrm{L}-12 \mathrm{~mm}, \mathrm{~B}-5 \mathrm{~mm}$ ) dark brown, ornamented with whitish and blackish patches, bands etc, throughout, head horizontal, eyes little incised, finely faceted, centrally black, surrounded by coppery facets; antennae very small, hardly surpassing the fore leg, segment- 1 thickened, segment- 3 longest; pronotum globuler, dark brown in color, densely, finely punctate with spars whitish pubescence, 2 semicircular black patches of pubescence prominently marked on either side of the pronotum at the middle; elytra dark
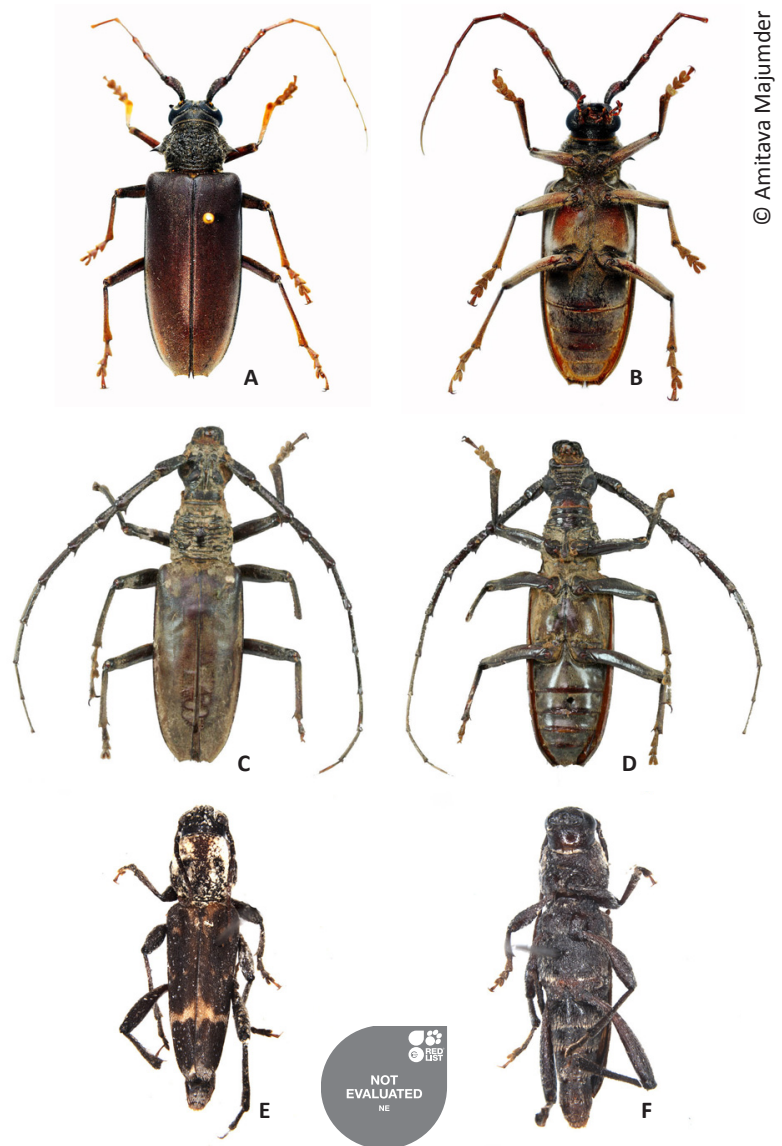

Image 1. Dorsal and ventral view of: A-B - Neoplocaederus ferrugineus (Linnaeus); C-D - Hoplocerambyx spinicornis (Newman); E-F - Xylotrechus smei (Laporte \& Gory).

brown, pubescence throughout, basal region with off white pubescence, coming downwards along the sutural margin, another 2 longitudinal bands started behind the scutellum forwarding towards apex, gradually out curved towards lateral margin at the middle of elytra, 2 wavy pale whitish bands on either side of elytra just behind the hind leg, 2 more irregular shaped whitish patches of pubescence on the lateral margin of the basal region, apex substrate, lateral angle with small spine; femur dumbbell shaped, tarsal claws more than $90^{\circ}$.

Distribution: India: Madhya Pradesh (Damoh), Chhattisgarh, Maharashtra, Odisha and Sikkim; Afghanistan, Bangladesh, Bhutan, Nepal.

\section{Tribe Callidiopini}

\section{Stenodryas apicalis (Gahan) (Image 2A,B)}

1893. Nyphasia apicalis Gahan, Ann. Mag. Nat. Hist.

(6) $11: 378$

1984. Stenodryas apicalis; Holzschuh, Ent. Basiliensa 9: 347. 
Material examined: Reg. no. 20093/H4A, 17.vi.2003, 1ex, Churna FRH, Pachmarhi Biosphere Reserve, Dist. Hoshangabad, Coll. K. Chandra..

Diagnostic characters: Small sized (L - 16mm, B $5 \mathrm{~mm}$ ), bright red brown in color, head, pronotum, legs and scape more darker, head horizontal; eyes large, black, upper lobe much smaller than lower one; antenna11 segmented, longer than body, segment-1 and -2 dark brown, rest black, segment- 1 small, dumbbell shaped, segment -3 longer than segment- 1 , segment 3 to 6 apically broad with acute spine on the inner margin, rest devoid of spines; pronotum globular, longer than broad, dark brown with black faint patches, lateral margins irregular, densely punctate; elytra dark brown, elongated, slender, apex charcoal black color, few fine longitudinal ridges throughout elytra; legs dark brown, basal region of the femur slender, abruptly bulged at the apex, tarsal claws less than $90^{\circ}$ angle.

Distribution: India: Madhya Pradesh (Hoshangabad), Chhattisgarh, Maharashtra and West Bengal; Myanmar, Nepal.

\section{Subfamily: Lamiinae}

Tribe: Ancylonotini

\section{Palimna annulata (Olivier) (Image 2C,D)}

1792. Cerambyx annulatus Olivier, Ene. Meth. Ins., 7: 465

1795. Cerambyx (Lamia) annulatus; Olivier, Ent. 4 (67): 95.

1817. Lamia Nodulosa Schönherr, Scaris, Lewerentziana 1 (3): 395. (syn.)

1856. Golsinda tesselata Pascoe, Trans. Ent. Soc. Lond. (Serie 2) 4: 49. (syn.)

1856. Golsinda reticulata Pascoe, Trans. Ent. Soc. Lond. (Serie 2) 4: 50. (syn.)

1866. Palimna Mouhotii Pascoe, Proc. Zool. Soc. Lond. 44: 237. (syn.)

1941. Palimna annulata; Beeson, Government of India, New Delhi : 198.

Material examined: Reg. no. 20094/H4A, 22.i.2003, 1ex, Mukki FRH, Kanha National Park, Dist. Balaghat, Coll. M. Limje..

Diagnostic characters: Body medium size ( $\mathrm{L}-20 \mathrm{~mm}$, B - $11 \mathrm{~mm}$ ), dull black, robust, elongate, ornamented with bands, patches and spots of dull yellow pubescence, head vertical, clypeus glossy brown, wide, transverse, band like, bare, frons elongate, rectangular parallel sided, clothed with dense yellow pubescence extending up to the middle of anterior lobe of eyes, rest bare, excepting a pair of small patches of yellow pubescence located on either side of the mid- longitudinal carina
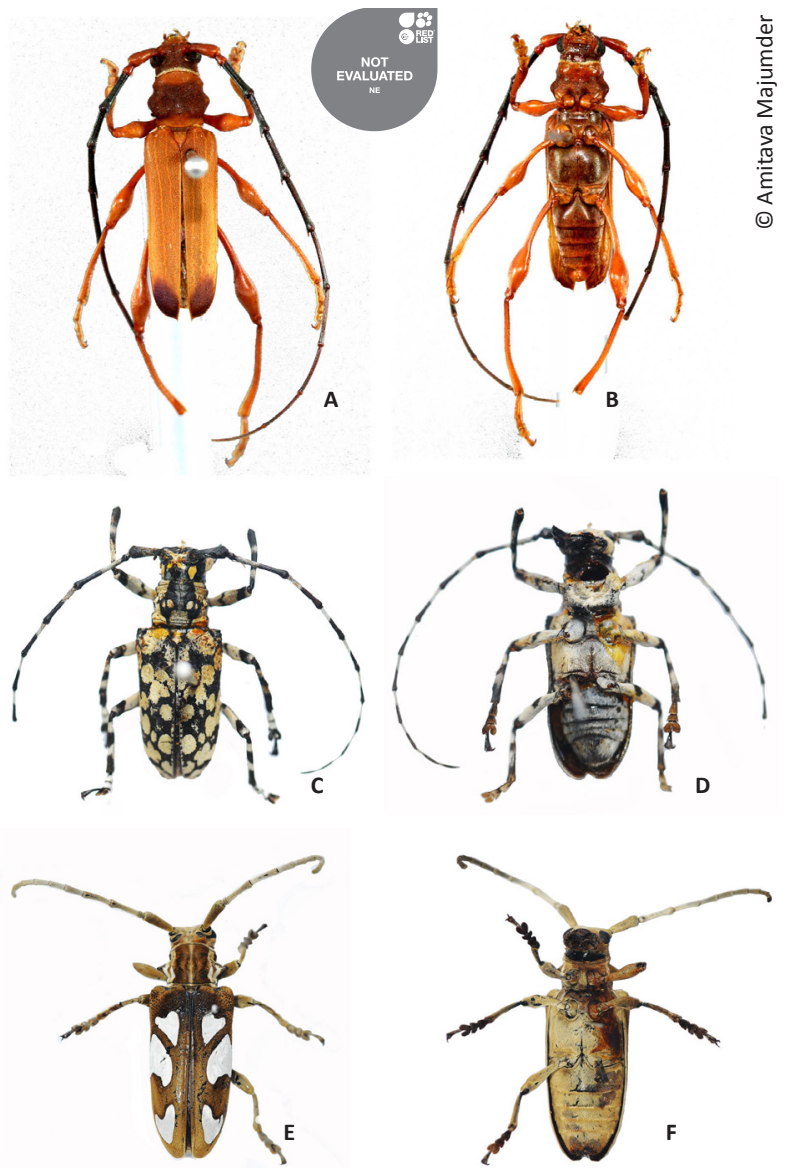

Image 2. Dorsal and ventral view of: A-B - Stenodryas apicalis (Gahan); C-D - Palimna annulata (Olivier); E-F - Calothyrza sehestedii (Fabricius).

extending from the base up to the vertex, strongly rogues and warty, coarsely punctuate; gena dark brown, large, densely, thickly clothed with similar pubescence, vertex wide, depressed at the bases of antenna, with band of dull yellow pubescence along the posterior lobe of eyes, two more spots of similar pubescence below the eye bar medially with a broad area marked by silvery white pubescence, few warts evident along the outer margin of the posterior lobe of eyes, besides few brown erect hairs distinct along the warts; eyes black, large, finely faceted, bar broad, anterior lobe large; antenna 11-segmented, much longer than body, all the segments basally white, apically dark brown excluding segment-1, segment-11 wholly dark brown, except segment -2 , all the segments widened apically and thickened with pubescence, segment -7 apically with tuft of pubescence, segment-1 small, robust, prominent scar mark at apex, segment-3 longest; pronotum elongate, transverse, both margins constricted; scutellum elongate broadly ' $U$ ' shaped, dark 
brown with dull yellowish pubescence, antero-lateral angles strongly warty; elytra basally brown, elongate, gradually narrowed towards apex, decorated with dull yellowish irregular, broad patches, apex truncated, disc basally with two pairs of blunt spinous projections, close to the suture, shoulder hump strongly marked by warts and blunt tubercles, coarsely punctate, these gradually effaced near apex, distal half strongly ridged, laterally margined, spines absent; venter red brown, silky dull yellow pubescence, denser laterally, coxal cavities open, prosternal plate narrow, widened basally, depressed in between the coxae, two bright yellow patches on fore coxae, mesosternal plate wide, rectangular, basal margin straight, laterally angulate, almost at the height of coxae, metepisternum broad, clothed with dense rusty yellow pubescence; legs black, robust, elongate, densely clothed with dull yellow pubescence leaving few bare areas marked by patches of black, fore tibia with a spine distally, warty, mid tibia with a pre-apical 'rainure', $3^{\text {rd }}$ tarsal segment bilobed, claws more than $90^{\circ}$ angle.

Distribution: Known from India, presently recorded from Madhya Pradesh (Balaghat); Borneo, Cambodia, China, Indonesia, Laos, Malaysia, Myanmar, Thailand.

\section{Tribe Phrynetini}

\section{Calothyrza sehestedii (Fabricius) (Image 2E,F)}

1798. Lamia Sehestedii Fabricius, Sup. Ent. Syst. Proft \& Storch, hafniae : 146.

1937. Calothyrza sehestedti; Breuning, Novitates Entomologicæ, 3ème sup. (46-49): 299.

Material examined: Reg. no. 20095/H4A, 12.vii.2006, 1 ex, Madan Mahal, Dist. Jabalpur, Coll. K. Chandra.

Diagnostic characters: Body medium size ( $\mathrm{L}$ $32 \mathrm{~mm}, \mathrm{~B}-12 \mathrm{~mm}$ ), yellowish brown, densely pubescent throughout, ornamented with whitish band and patches, head vertical, yellowish brown pubescence with dark brown patches, frons sub- squarish surface smooth, clypeus small, dark brown, eyes sub-divided, lower one much larger than upper one, vertex finely incised in between the eyes, dark brown patches of pubescence in between eyes and anterior margin of pronotum; antenna 11- segmented, stout, densely pubescent, segment- 1 robust stout, segment- 3 larger than segment- 1 and 4, 5 to 11 segments smaller; pronotum elongated, subsquarish, dark brown pubescence throughout, broad, distinct elongated band of whitish pubescence on the lateral margins, slender elongated faint patch on middle, connected the anterior and posterior margins of pronotum, lateral margin with strong tubercles; scutellum broadly tongue shaped; elytra elongated, clothed with brownish to dark brownish patches of pubescence, ornamented with three distinct silky whitish patches: 1 heart shaped close to sutural margin, nearer to basal region on elytra, second one largest among the three, nearest to lateral margin and hind femur, the third one broadly ' $V$ ' shaped in between the sutural and lateral margin, very close to apex, apex of the elytra sub rounded, venter brown to dark brown in color, coxal cavities closed, meso- sternal process broad apically truncated, legs stout, femur robust, tibia elongated, tarsal claw not more than $90^{\circ}$ angle.

Distribution: India: Himalayas, Madhya Pradesh (Jabalpur); Sri Lanka.

\section{Tribe Apomecynini}

\section{Apomecyna histrio (Fabricius) (Image 3A,B)}

1793. Lamia histrio Fabricius, Ent. Syst. emendata et aucta. 1 (2), 1-538: 288.

1802. Saperda alboguttata Megerle, Cat. Insect. quae Viennae Austriae: 10. (syn.)

1840. Apomecyna histrio; Castelnau, Histoire Naturelle des Insectes Coléoptères, Paris: 492.

1868. Apomecyna quadrifasciata Thomson, Physis Rec. Hist. Nat., Paris 2 (6): 159. (syn.)

Material examined: Reg. no. 20096/H4A, 1.vii.2001, 1ex, Karmajhiri, Pench Tiger Reserve, Dist. Seoni, Coll. K. Chandra.

Diagnostic characters: Small size, (L - 10mm, B $4 \mathrm{~mm}$ ) dark brown, elongated, decorated with white spots, head almost rounded in shape, covered with dense yellowish brown pubescence; eyes divided in two parts, upper lobe smaller than the lower lobe, eyes finely faceted black in colour, gena sub-quadrate, frons subquadrate; antennae black, hardly extending up to the middle of the elytra, covered with yellowish pubescence, $3^{\text {rd }}$ and $4^{\text {th }}$ segment of the antennae is larger, $5^{\text {th }}$ to $11^{\text {th }}$ segments small, equal in size; pronotum cylindrical, covered with brown pubescence and punctures; pronotum decorated with white patches, 2 laterals on mid dorsal line and other 2 longitudinally at the apex of pronotum, elytra elongated, apex not fully rounded, covered with yellowish brown pubescence; elytra decorated with longitudinal transverse white rounded patch, patches are variable in number, patches arranged in four transverse bands, first and last bands are small, usually composed with two patches; elytra covered with dense longitudinal punctures; scutellum tongue shaped covered with yellowish brown pubescence.

Distribution: India: Madhya Pradesh (Seoni), Chhattisgarh and Tamil Nadu; Bangladesh, Borneo, China, Indonesia, Japan, North Korea, Laos, Malaysia, Nepal, Pakistan, Philippines, Russia, Taipei. 

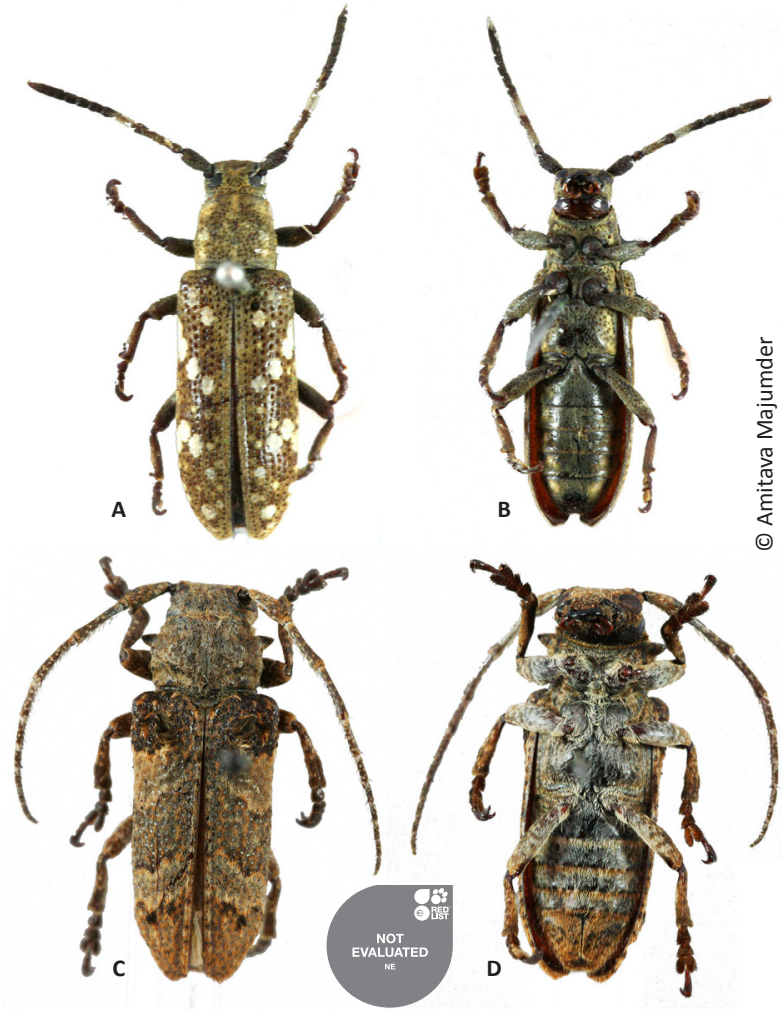

Image 3. Dorsal and ventral view of: A-B - Apomecyna histrio (Fabricius); C-D - Niphona fuscatrix (Fabricius).

\section{Tribe Pteropliini}

\section{Niphona fuscatrix (Fabricius) (Image 3C,D)}

1793. Lamia fuscator Fabricius, Ent. syst. eme. et aucta. Hafniae, Proft. 1(2): 291.

1858. Nyphona cylindracea White, Ann. Mag. Nat. Hist., London (3) 2: 268. (syn.)

1891. Ocheutes spinicollis Bates, The Entomologist. 24: 22. (syn.)

1962. Niphona (Niphona) fuscatrix; Breuning, Ent. Arbeiten aus dem Museum G. Frey, Tutzing bei München 13 (2): 399.

Material examined: Reg. no. 20097/H4A, 25.vii.1999, 1ex, Mohania, Dist. Sidhi, Coll. K.Chandra..

Diagnostic characters: Body small in size, $(\mathrm{L}-17 \mathrm{~mm}$, B - $6 \mathrm{~mm}$ ) dark brown, ornamented with yellowish-brown patches, head small vertical, frons broad sub-squarish, densely pubescent, vertex broad in between the eyes; eyes strongly sub-divided, lower lobe much larger than upper one; antenna 11- segmented, dark brown with yellowish patches of pubescence, inner margin with long hairs, segment 1 small, robust, segment 4 little longer than segment 3 , much longer than segment 1 ; pronotum broader than long, globuler, surface roughen with ridges, densely pubescence, upper surface of the lateral margin medially out curved, lower surface with two prominent tubercles one big and other one small; scutellum small broadly $U$ shaped; elytra parallel sided, ornamented with 2 broad brown patches of pubescence, one in basal region and second one at the middle, deeply largely punctured more in basal region, humeral angle prominent, hairiness more on basal region, 2 long erected patches of brown hairs on either side of sutural margin, 4 small erected brown patches of hairs on either side of the sutural margin close to apical region, declivity on the epical region on elytra, elytral apex somewhat sub rounded; venter dark brown clothed with yellowish to off white pubescence, mesosternal plate slightly raised at the anterio, basal margin substrate, femur stout, tarsal claw less than $90^{\circ}$ angle.

Distribution: India: Odisha, Madhya Pradesh (Sidhi) and Tamil Nadu; Sri Lanka.

\section{Discussion}

Except for a few notable literatures, proper taxonomic and distributional studies on this economically important insect group are lacking in India. The present work may be considered as the first consolidated account of Cerambycidae of Madhya Pradesh. Altogether, 18 species of longhorn beetles are reported in the present paper. Among them eight species have been collected from different parts of Madhya Pradesh during the present survey by the scientists of the Zoological Survey of India from 1999 to 2011. Of them seven species are reported for the first time from Madhya Pradesh. All the seven species reported here have extended distribution up to the Palaearctic Region except two species, namely, Stenodryas apicalis (Gahan) and Niphona fuscatrix (Fabricius), which are restricted to the Oriental region. All these species are commonly distributed within India, except Neoplocaederus ferrugineus (Linnaeus) reported from Tamil Nadu and Apomecyna histrio (Fabricius) from Tamil Nadu and Chhattisgarh other than Madhya Pradesh.

India having a mosaic of varied climate and habitat, proper studies on distribution of flora and fauna, especially of these ecologically important insects, are essential for effective implication of conservation strategies.

\section{REFERENCES}

Basak, P.K. \& S. Biswas (1993). Insecta: Coleoptera: Cerambycidae, pp.185-195 In: Fauna of Orissa. State Fauna series. Zoological Survey of India, Kolkata, India.

Biswas, S. \& P.K. Basak (1992). Studies on Longicorn beetles (Coleoptera: Cerambycidae) from India Part I. On Indian species Apomecyna 
Latreille with a key to Indian genera of tribe Apomecynini. Records of the Zoological Survey of India 92(1-4): 161-172.

Chandra, K., R.M. Sharma \& P. Ojha (2010). A compendium on the faunal resources of Narmada Basin in Madhya Pradesh. Records of the Zoological Survey of India. Occasional paper 310: 57.

Delahaye, N. (2014). Private Communication. http://prioninae.org

Lamiaires du Monde (2013). Electronic database accessible at http:// www.lamiinae.org/. Accessed on 23 May 2013.

Gahan, C.J. (1906). The Fauna of British India including Ceylon and Burma. Coleoptera: Cerambycidae. Taylor \& Francis, London. 329p.

Ghate, H.V. (2012). Insecta: Coleoptera: Cerambycidae, pp.503-505. In Fauna of Maharashtra, State Fauna Series 20 (Part- 2). Zoologica Survey of India, Kolkata, India.

Gressitt, J.L., J. A. Rondon \& S. Breuning (1970). Cerambycid beetles of Laos. Pacific Insect Monograph 24: 1-651.

Khan, T.N. \& T. Khan (1987). Bology of Nyphasia apicalis Gahan (Coleoptera: Cerambycidae) with particular emphasis on emergence and imaginal life. Proceedings of Indian Academy of Science, Animal Sciences 96(4): 403-415.
Makihara, H., A. Mannakkara, T. Fujimura \& A. Ohtake (2008). Checklist of longicorn coleoptera of Sri Lanka. Vesperidae and Cerambycidae excluding Lamiinae. Bulletin of FFPRI 7(2): 95-110.

Mukhopadhyay, P. \& S.K. Halder (2003). Insecta: Coleoptera: Cerambycidae, pp. 181-199. In: Fauna of Sikkim, State Fauna Series 9 (Part 3). Zoological Survey of India, Kolkata, India.

Mukhopadhyay, P. \& S. Biswas (2000). Coleoptera: Cerambycidae, pp.41-67. In: Fauna of Meghalaya, State Fauna Series 4 (Part 5). Zoological Survey of India, Kolkata, India.

Namboodiri, M.P.S. \& G. Thirumalai (2009). Insecta: Coleoptera: Cerambycidae, pp.91-97. In: Fauna of Tamil Nadu, State Fauna Series 17 (Part-1). Zoological Survey of India, Kolkata, India.

Sama, G. (1991). Note sulla nomenclatura dei Cerambycidae della regione mediterranea (Coleoptera). Bollettino della Società Entomologica Italiana 123(2): 121-1. 